\section{OPEN ACCESS}

Edited by:

Peter R. Hobson,

Queen Mary University of London,

United Kingdom

Reviewed by:

Fabrizio Ambrosino,

University of Campania Luigi Vanvitelli,

Francesco Nozz

University of Trento, Italy

${ }^{*}$ Correspondence:

F. Cerutti

francesco.cerutti@cern.ch

Specialty section:

This article was submitted to

Radiation Detectors and Imaging,

a section of the journal

Frontiers in Physics

Received: 01 October 2021 Accepted: 08 November 2021

Published: 27 January 2022

Citation:

Ahdida C, Bozzato D, Calzolari D, Cerutti F, Charitonidis N, Cimmino A,

Coronetti A, D'Alessandro GL,

Donadon Servelle A, Esposito LS,

Froeschl R, García Alía R,

Gerbershagen A, Gilardoni $S$,

Horváth $D$, Hugo $G$, Infantino $A$, Kouskoura $V$, Lechner $A$, Lefebvre $B$,

Lerner G, Magistris M, Manousos A,

Moryc G, Ogallar Ruiz F, Pozzi F,

Prelipcean D, Roesler S, Rossi R,

Sabaté Gilarte M, Salvat Pujol F,

Schoofs $P$, Stránský $V$, Theis $C$,

Tsinganis A, Versaci $R$, Vlachoudis $V$, Waets A and Widorski M (2022) New

Capabilities of the FLUKA Multi-

Purpose Code.

Front. Phys. 9:788253.

doi: 10.3389/fphy.2021.788253

\title{
New Capabilities of the FLUKA Multi-Purpose Code
}

\section{Ahdida ${ }^{1}$, D. Bozzato ${ }^{1,2}$, D. Calzolari ${ }^{1}$, F. Cerutti ${ }^{1 *}$, N. Charitonidis ${ }^{1}$, A. Cimmino ${ }^{3}$, A. Coronetti ${ }^{1,4}$, G. L. D'Alessandro ${ }^{1}$, A. Donadon Servelle ${ }^{1,5}$, L. S. Esposito ${ }^{1}$, R. Froeschl ${ }^{1}$, R. García Alía ${ }^{1}$, A. Gerbershagen ${ }^{1}$, S. Gilardoni ${ }^{1}$, D. Horváth ${ }^{3}$, G. Hugo ${ }^{1}$, A. Infantino ${ }^{1}$, V. Kouskoura ${ }^{1}$, A. Lechner ${ }^{1}$, B. Lefebvre ${ }^{3}$, G. Lerner ${ }^{1}$, M. Magistris ${ }^{1}$, A. Manousos ${ }^{1,6}$, G. Moryc ${ }^{1}$, F. Ogallar Ruiz ${ }^{1,7}$, F. Pozzi ${ }^{1}$, D. Prelipcean ${ }^{1,8}$, S. Roesler ${ }^{1}$, R. Rossi ${ }^{1}$, M. Sabaté Gilarte ${ }^{1}$, F. Salvat Pujol ${ }^{1}$, P. Schoofs ${ }^{1}$, V. Stránský ${ }^{3}$, C. Theis ${ }^{1}$, A. Tsinganis ${ }^{9}$, R. Versaci ${ }^{3}$, V. Vlachoudis ${ }^{1}$, A. Waets $^{1}$ and M. Widorski ${ }^{1}$}

${ }^{1}$ European Organization for Nuclear Research (CERN), Geneva, Switzerland, ${ }^{2}$ Karlsruhe Institute for Technology (KIT), Karlsruhe, Germany, ${ }^{3} \mathrm{ELI}$ Beamlines Centre, Institute of Physics, Czech Academy of Sciences, Dolní Břežany, Czech Republic, ${ }^{4}$ Department of Physics, University of Jyväskylä, Jyväskylä, Finland, ${ }^{5}$ Ecole Polytechnique Fédérale de Lausanne, Institute of Physics, Lausanne, Switzerland, ${ }^{6}$ Department of Physics, Aristotle University of Thessaloniki, Thessaloniki, Greece, ${ }^{7}$ Department of Atomic, Molecular and Nuclear Physics, University of Granada, Granada, Spain, ${ }^{8}$ Department of Physics, Technical University of Munich (TUM), Munich, Germany, ${ }^{9}$ European Commission, Joint Research Centre (JRC), Geel, Belgium

FLUKA is a general purpose Monte Carlo code able to describe the transport and interaction of any particle and nucleus type in complex geometries over an energy range extending from thermal neutrons to ultrarelativistic hadron collisions. It has many different applications in accelerator design, detector studies, dosimetry, radiation protection, medical physics, and space research. In 2019, CERN and INFN, as FLUKA copyright holders, together decided to end their formal collaboration framework, allowing them henceforth to pursue different pathways aimed at meeting the evolving requirements of the FLUKA user community, and at ensuring the long term sustainability of the code. To this end, CERN set up the FLUKA.CERN Collaboration ${ }^{1}$. This paper illustrates the physics processes that have been newly released or are currently implemented in the code distributed by the FLUKA.CERN Collaboration ${ }^{2}$ under new licensing conditions that are meant to further facilitate access to the code, as well as intercomparisons. The description of coherent effects experienced by high energy hadron beams in crystal devices, relevant to promising beam manipulation techniques, and the charged particle tracking in vacuum regions subject to an electric field, overcoming a former lack, have already been made available to the users. Other features, namely the different kinds of low energy deuteron interactions as well as the synchrotron radiation emission in the course of charged particle transport in vacuum regions subject to magnetic fields, are currently undergoing systematic testing and benchmarking prior to release. FLUKA is widely used to evaluate radiobiological effects, with the powerful support of the Flair graphical interface, whose new generation (Available at http://flair.cern) offers now additional capabilities, e.g., advanced 3D visualization with photorealistic rendering and support

\footnotetext{
${ }^{1}$ In this paper, depending on the context, references to FLUKA are to code developed jointly by INFN and CERN until 31 August 2019, or to further code developments performed after this date in the framework of the FLUKA.CERN Collaboration ${ }^{2}$ At https://fluka.cern.
} 
for industry-standard volume visualization of medical phantoms. FLUKA has also been playing an extensive role in the characterization of radiation environments in which electronics operate. In parallel, it has been used to evaluate the response of electronics to a variety of conditions not included in radiation testing guidelines and standards for space and accelerators, and not accessible through conventional ground level testing. Instructive results have been obtained from Single Event Effects (SEE) simulations and benchmarks, when possible, for various radiation types and energies. The code has reached a high level of maturity, from which the FLUKA.CERN Collaboration is planning a substantial evolution of its present architecture. Moving towards a modern programming language allows to overcome fundamental constraints that limited development options. Our long term goal, in addition to improving and extending its physics performances with even more rigorous scientific oversight, is to modernize its structure to integrate independent contributions more easily and to formalize quality assurance through state-of-the-art software deployment techniques. This includes a continuous integration pipeline to automatically validate the codebase as well as automatic processing and analysis of a tailored physics-case test suite. With regard to the aforementioned objectives, several paths are currently envisaged, like finding synergies with Geant4, both at the core structure and interface level, this way offering the user the possibility to run with the same input different Monte Carlo codes and crosscheck the results.

Keywords: FLUKA, Monte Carlo transport, beam-matter interaction, high energy physics, crystal channeling, medical physics, single event effects (SEE)

\section{INTRODUCTION}

The FLUKA (FLUktuierende KAskade in German, i.e., fluctuating cascade) code was born at the European Organisation for Nuclear Research (CERN) from the work of J. Ranft, who in the mid nineteen-sixties developed several Monte Carlo programs for the determination of shielding thicknesses, estimation of induced radioactivity levels and prediction of dose absorption in critical components at high energy proton accelerators. As one of them, FLUKA was a purely analogue code simulating the particle cascade in a cylindrical block of a specified elemental material and calculating the spatial distribution of nuclear reaction ("star") and energy deposition densities per incoming primary proton or charged pion. The user input was to be provided by means of formatted control and data cards, representing a lasting legacy.

Building on that ground, the first generation of FLUKA versions spanned until the late seventies, relying again on the work of J. Ranft (then affiliated with the Karl Marx University of Leipzig) and his collaboration with J. Routti of Helsinki University of Technology (HUT). A second generation, addressing the reformulation of the hadron interaction model among other significant advances, can be identified across the eighties with the joint effort of CERN (G.R. Stevenson and A. Fassò), HUT (J. Sandberg and P. Aarnio) and the Leipzig University group (J. Ranft and H.-J. Moehring).

The modern (third generation) FLUKA moved from a plan by A. Ferrari (initially INFN, then CERN) and A. Fassò to transform the code into a multi-purpose multi-particle tool and featured a substantial restructuring, improvement and extension, eventually increasing by one order of magnitude the Fortran source size, with the contribution of P. Sala (INFN). In 2000, dedicated support was provided by NASA to enable the treatment of heavy ion interactions, including the interface to external event generators. In 2003, a collaboration agreement between CERN and INFN was set up in order to develop, maintain and release FLUKA, integrating through the years the work of several other collaborators (see for instance [1] and references therein).

In 2019, more than 15 years on, the formal collaborative framework between the two copyright holders was succeded by an arrangement aimed at ensuring FLUKA's long-term sustainability and capability to meet the evolving requirements of its user community. This offered the opportunity to leverage lessons learned and current standards in the organization of software collaborations, while fostering the active involvement of both established FLUKA contributors as well as new partners. As a result, CERN recently put in place a multilateral collaboration framework, already joined by ELI Beamlines, and a new licensing scheme expanding the access to the source code and allowing for its customization as well as result intercomparison and physics model benchmarking. In parallel, a modern user support platform has been adopted $^{3}$ and a significantly higher distribution pace has materialized, with

${ }^{3}$ https://fluka-forum.web.cern.ch/ 


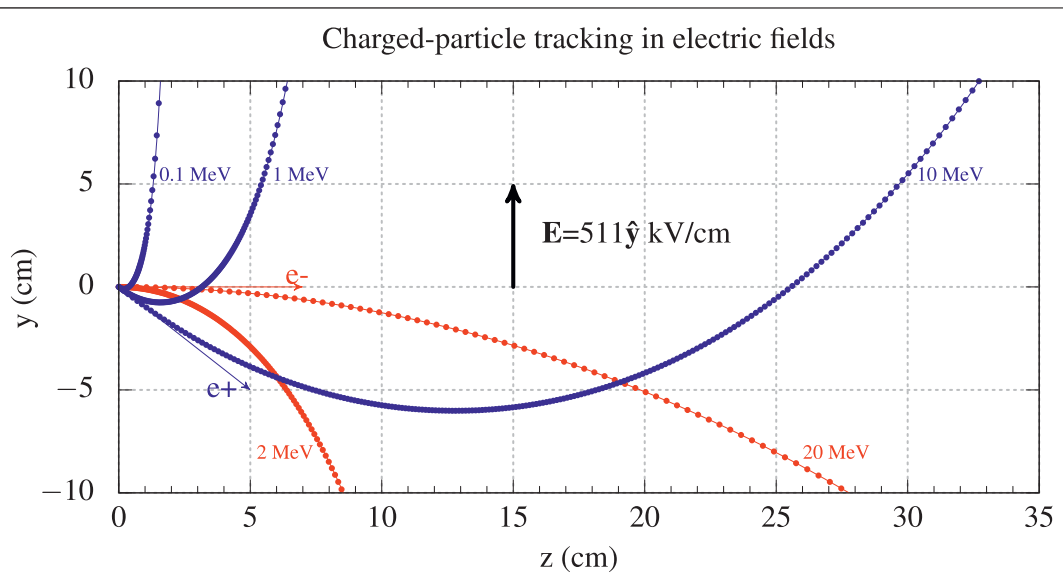

FIGURE 1 | Comparison between FLUKA (dots) and analytical (solid curves) electron (red) and positron (blue) trajectories starting at the origin of coordinates along the direction indicated by the respective arrows, in a $511 \mathrm{kV} / \mathrm{cm}$ electric field along the $y$ axis for energies ranging from 0.1 to $20 \mathrm{MeV}$ as per the figure labels.

two major releases in 2020 and a third one planned to take place before the end of 2021 .

Selected physics developments that provide FLUKA with new capabilities are detailed in section 2. They are the description of coherent effects experienced by charged particles traversing crystals, the transport of charged particles in electric fields, the simulation of nuclear reactions by deuterons below $300 \mathrm{MeV}$, and the synchrotron radiation emission by charged particles in magnetic fields.

Section 3 reports on advances of the FLUKA Advanced InteRface (Flair), whose restructuring in Python v3 integrated a new medical visualization module highly improving the volumetric rendering of voxelized phantoms and dose distributions. Section 4 focuses on another field of FLUKA applications, namely radiation to electronics, and, after reviewing relevant achievements of the last decade, includes new results regarding photon-induced Single Event Effects (SEE).

Section 5 discusses the main quality assurance measures featuring the development, testing and benchmarking framework put in place for FLUKA v4 (that is currently distributed by the FLUKA.CERN Collaboration as fourth FLUKA generation) in order to properly validate the code evolution prior to release and indicate the improvement needs. Finally, section 6 introduces the long term perspective and the associated project stages towards a fifth FLUKA generation, the first of which already started in parallel with the steady investment on FLUKA v4.

\section{NEW PHYSICS DEVELOPMENTS}

\subsection{Transport of Charged Particles in Electric Fields}

FLUKA has been able to track charged particles in magnetic fields since the late 1980s, thus allowing users from a relatively early stage on to model charged particle transport e.g. in complex particle accelerator components, such as dipole or quadrupole magnets and beam kickers. The availability of user

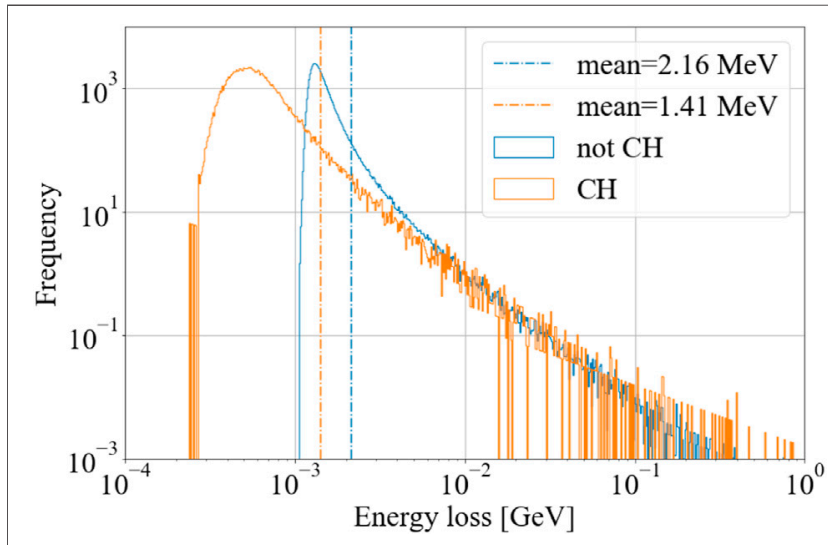

FIGURE 2 | Energy loss distribution for $180 \mathrm{GeV}$ protons on a $4 \mathrm{~mm}$ long crystal in channeling regime (orange) and in amorphous regime (blue).

routines provides full customization capabilities to tackle the transport of charged particles in arbitrary user-defined magnetic fields.

Instead, the transport of charged particles in electric fields [2] was a longstanding pending ingredient in FLUKA's transport algorithm. This lack prevented users from modelling electrostatic septa in particle accelerator extraction studies, for example. Users were forced to resort to a proxy magnetic field effectively providing the desired force (permissible whenever the change of the charged particle energy is negligible). To overcome this shortcoming, a dedicated module for charged particle transport in electric fields in vacuum has been recently included in FLUKA.

The transport of charged particles in both magnetic and electric (static) fields relies on the same adaptive stepping algorithm to solve the resulting equation of motion, offering users an accurate control of both the particle step size and the tolerance with which boundaries to nearby regions can be intercepted by the (effectively curved) particle steps. FLUKA's module for charged particle tracking in electric fields accounts for 

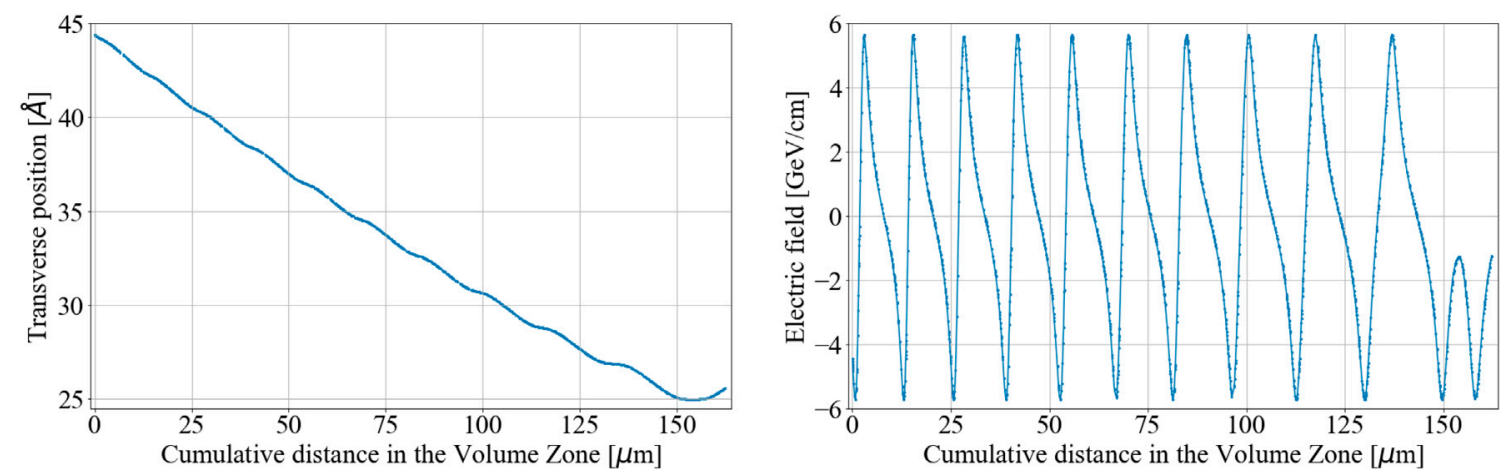

FIGURE 3 | Left: Quasi-channeled particle trajectory through a crystal close to the tangency point as a function of the cumulative longitudinal coordinate. Oscillations are due to the Molière potential and volume reflection can be seen at the right-hand side of the chart. Right: Corresponding electric field along the particle trajectory.

the variation (decrease or increase) of the particle's kinetic energy, and it includes an algorithm which treats situations where the electrostatic force opposes the direction of motion, accurately resolving the trajectory turning point where relevant.

Following the example proposed by A. Bielajew [3], Figure 1 displays the excellent agreement between the analytical (solid curves) and FLUKA (symbols) electron (red) and positron (blue) trajectories starting at the origin of coordinates along the direction indicated by the respective arrows in the yz plane, in an electric field of $511 \mathrm{kV} / \mathrm{cm}$ along the $y$ axis.

\subsection{Coherent Effects in Crystals}

Coherent interactions of charged particles in bent crystals can be used for beam collimation or extraction purposes. Crystals have long been under study at CERN and in other research centers [4, 5] leading to very promising results for the Large Hadron Collider (LHC) collimation [6, 7]. In this context, crystals are planned to be part of the collimation system for High Luminosity LHC Ion runs [8]. In parallel with this activity, a module able to reproduce the various coherent effects such as channeling, volume capture and volume reflection was added to FLUKA [9]. This module also accounts for the change in both interaction and energy loss rate in these regimes. In the following, relevant aspects of the model are introduced and the most recent developments are detailed.

In a FLUKA geometry, a crystal is a region for which several characteristics such as torsion, temperature, radius of curvature and channel orientation are specified. These are set up independently and it is therefore the user's responsibility to ensure that microscopic features of the device are consistent with its geometry. However, this decoupling offers an additional degree of freedom, for instance the possibility to introduce a deliberate mismatch between crystal edges and plane orientation to reproduce miscut ${ }^{4}$.

${ }^{4}$ Miscut is a crystal manufacturing defect causing crystal edges to not be perfectly parallel to the channel planes. More information on miscut and its effects on channeling can be found in e.g., [11].

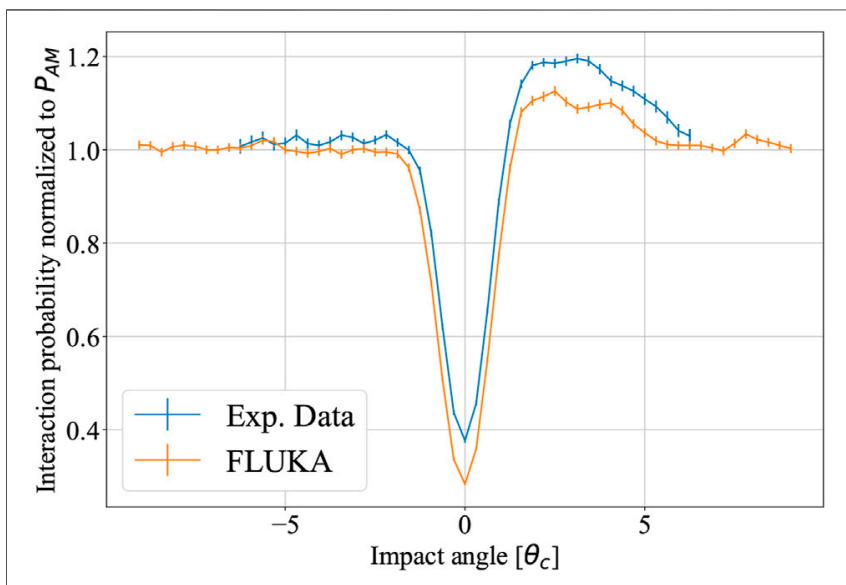

FIGURE 4 | Nuclear interaction rate as a function of the particle incoming angle, normalized to the interaction rate in amorphous mode. The dip corresponds to channeled particles, experiencing low interaction rate in the channel. The excess observed for small positive angles corresponds to the increased rate experienced by quasi-channeled particles when crossing multiple channels. Experimental data from [13].

As the crystal channel direction is defined, the incoming angle of each entering particle can be calculated. When the angle is small enough, the particle becomes trapped in between the transverse Molière potential barriers sitting at each atomic center. The particle enters the channeling regime and is transported along the channel. For a bent crystal, this implies tracking along an arc which is implemented through the use of a proxy magnetic field. In addition, the channeled particle oscillates transversally between the channeling planes with an amplitude depending on its transverse momentum.

This oscillation is important for the computation of reduced interaction rate in channeling. Firstly, the electronic stopping power for channeled projectiles is reduced compared with the one of an amorphous medium. The FLUKA algorithm uses a prescription similar to that of Esbensen et al. [10], maintaining the contribution of distant 
collisions to the stopping power unaltered and scaling that of close collisions ${ }^{5}$ by the fraction of the channel electron density subtended by the oscillating trajectory of the particle. The reduction factor is typically of the order of 2 , as shown in Figure 2 for the case of $180 \mathrm{GeV}$ protons through a $4 \mathrm{~mm}$-long Si crystal.

Secondly, Coulomb scattering of channeled particles on the screened potential of target atoms is computed on a single event basis rather than in multiple scattering. Contributions from small impact parameter collisions are suppressed, with a cutoff corresponding to the minimum distance between the particle oscillatory path and the atomic centers.

Thirdly, nuclear interactions are scaled to the average nuclear density along the particle oscillatory path. This leads to a substantial reduction of the nuclear interaction rate [11] for the channeled population at large.

Coherent effects are not limited to channeling. Some particles can experience volume effects inside the crystal: a reflection on the crystal planes or a capture in their current channel after interaction. Both take place when the particle trajectory is almost tangent with the planes, which can only happen when their incoming angle has the same sign as the crystal bending. In order to reproduce these mechanisms, the equations of motion of the particle are solved in the potential of interest, in this case an electrostatic Molière potential to which a centrifugal term is added due to bending.

The volume reflection naturally results from this tracking: on Figure 3 the trajectory of a quasi-channeled particle is displayed close to its tangency point with the planes, as a function of the longitudinal coordinate. After crossing several planes, the particle acquires a small angular deflection in the direction opposite to bending. While progressing through this region, quasi-channeled particles can undergo a disrupting scattering event, which can lead to the capture of the particle if its transverse momentum drops below the Molière potential energy. A captured particle becomes channeled from that point on until it is dechanneled or reaches the end of the crystal.

Microscopic treatment of the coherent effects of quasichanneled particles is an important recent development of the model. The nuclear interaction rate is altered for quasichanneled particles, akin to what is implemented for channeled ones. However, quasi-channeled particles cross atomic planes and the resulting interaction rate is higher than in amorphous mode. This increase is apparent on Figure 4 displaying the nuclear interaction rate as a function of the incoming angle of the particle for a crystal bent towards positive angles. The main dip reflects the suppression of nuclear interactions for channeled particles. Next to it, the interaction rate comes back to amorphous regime values, with the excess on the right reflecting the increased interaction probability of quasi-channeled particles, in accordance with experimental data. The extent

${ }^{5}$ giving an otherwise equal contribution to the stopping power as per [12]. of the excess, however, remains slightly lower than the data shows and will be the focus of upcoming developments.

\subsection{Low-Energy-Deuteron Nuclear Interactions}

Another longstanding shortcoming of FLUKA's transport algorithm concerned the lack of a dedicated model for nuclear reactions of low energy deuterons (below $150 \mathrm{MeV} / \mathrm{n}$ ). Up to version 4.1, FLUKA included a coarse tentative model to effectively split the deuteron into its constituent neutron and proton, each taking $\sim 50 \%$ of the kinetic energy of the deuteron. FLUKA was therefore not recommended for deuteron-beam applications.

Recently, deuteron transport has gained increased attention, for applications as diverse as the production of radioisotopes for medical applications using deuteron beams [12, 13], their radiobiological effects and associated radiation protection aspects [14], as well as for nuclear fusion studies [15]. Furthermore, deuteron production from hadron- and nucleusnucleus inelastic interactions is often far from negligible; an accurate description of their subsequent nuclear interactions is necessary. Consequently, a dedicated model for low energy deuteron nuclear reactions has been developed and will be included in FLUKA 4.2.

The model consists of the following main components: deuteron stripping to the continuum is modelled on the basis of the Serber model [16] for the (uncorrelated) energy and angle distribution of the stripped nucleon, coupled to FLUKA's hadron-nucleus interaction model PEANUT (PreEquilibrium Approach to NUclear Thermalization) [17] in order to properly describe the nuclear inelastic interaction of the remaining nucleon with the target nucleus; deuteron elastic break-up is described within the zero-range distorted wave Born approximation (DWBA) for nuclei with atomic number $Z \geq 3$ [18]; deuteron stripping to discrete levels is also accounted for within the DWBA [19] for the $(\mathrm{d}, \mathrm{p})$ and $(\mathrm{d}, \mathrm{n})$ reaction channels on a selection of light isotopes for which this interaction mechanism is relevant, including ${ }^{6,7} \mathrm{Li}$, ${ }^{9} \mathrm{Be}$, and ${ }^{12} \mathrm{C}$. More details and benchmarking of this model will be provided in an upcoming dedicated publication.

Furthermore, a complete-fusion channel is included via the Boltzmann Master Equation (BME) event generator, already employed in FLUKA to describe nucleus-nucleus inelastic interactions up to $150 \mathrm{MeV} / \mathrm{n}$ [20]. Whereas the three model components described above imply at most one nucleon penetrating the nucleus, the complete-fusion channel effectively permits both nucleons to penetrate the target nucleus, thus providing a larger excitation energy and accounting for the experimentally observed emission of light nuclear fragments beyond a neutron or proton $\left(\mathrm{d}, \mathrm{t},{ }^{3} \mathrm{He}\right.$, ${ }^{4} \mathrm{He}$, etc.).

As an example showcasing the performance of the new low-energy deuteron nuclear interaction model in FLUKA, Figure 5 displays the double-differential (energy and angle) cross section for the emission of protons from ${ }^{58} \mathrm{Ni}$ under 


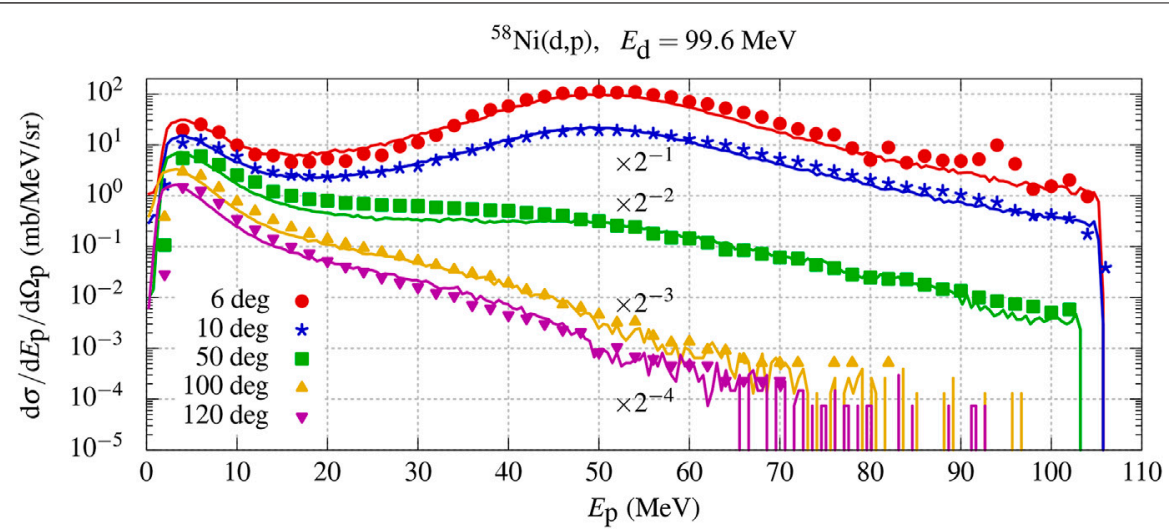

FIGURE 5 | Comparison in absolute units between FLUKA (solid curve) vs experimental (symbols) [21] double-differential (energy and angle) proton emission cross section from ${ }^{58} \mathrm{Ni}$ under bombardment with 99.6 MeV deuterons, resolved as a function of the proton energy $E_{\mathrm{p}}$ for the various indicated emission angles in the lab frame. The curve for 6 deg emission is in absolute units; subsequent curves have been scaled down respectively by the indicated factors for clarity.
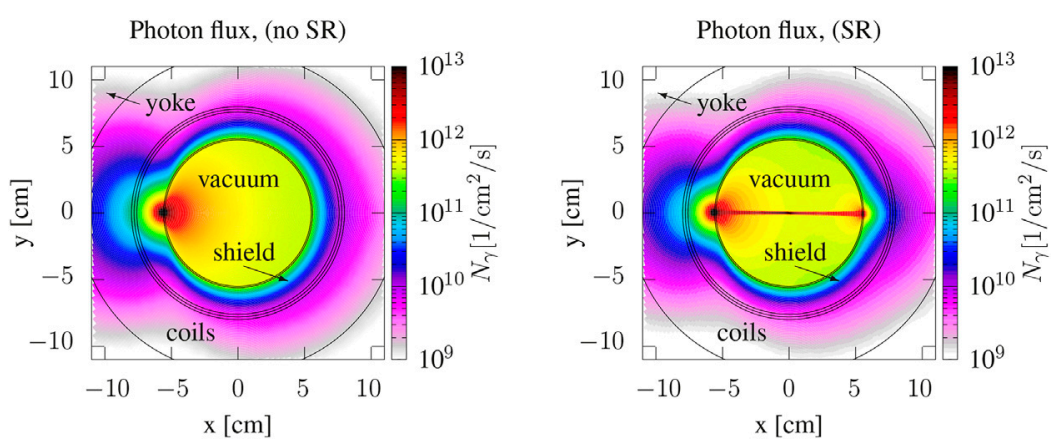

FIGURE 6 | Photon flux distribution in the 10.2 T bending dipole of a $4.5 \mathrm{~km}$ ring accomodating a 1.5 TeV negative muon beam with a bunch intensity of $2 \times 10^{12}$ particles (transverse section). Bunches are injected with a $12 \mathrm{~Hz}$ frequency. Sychrotron radiation emitted by high energy electrons generated by muon decay is neglected on the left and accounted for on the right.

99.6 $\mathrm{MeV}$ deuteron bombardment at various emission angles, resolved as a function of the proton energy, comparing in absolute units the FLUKA simulation results (solid curves) with available experimental data [21] (in symbols). The simulated proton energy spectra exhibit a remarkably good agreement with the experimental data, reproducing their essential features: a peak at half the deuteron kinetic energy, particularly intense for forward emission angles and due to deuteron stripping to the continuum, as well as a peak at low proton energies from the evaporation stage of the conjugate neutron-nucleus inelastic interaction. Figure 5 shows that good agreement is preserved throughout the angular domain covered by the experimental data.

\subsection{Synchrotron Radiation Emission During Charged Particle Tracking}

The emission of synchrotron radiation (SR) photons is possible in FLUKA by means of a special source term. This allows the user to define up to two arcs of a charged particle trajectory in a dipole field, along which SR photons are sampled as source particles for the simulation. The radiating charged particle itself is not explicitly tracked. Such a scheme is useful for applications where SR emission is dominated by beam particles, for example when modelling arcs of electron storage rings. This approach is however not suitable for cases where secondary and higher-generation particles lose a significant fraction of their energy through SR emission. This is for instance the case for high energy muon storage rings and colliders, where SR emission by decay electrons and positrons cannot be neglected when assessing the heat load distribution in magnets or the beaminduced background in detectors.

To overcome this drawback, the FLUKA tracking algorithm for charged particles in a magnetic field in vacuum has been enhanced by including the emission of SR photons as a supplementary discrete process during tracking.

SR photons are explicitly generated and the corresponding energy loss of the radiating charged particle is taken into account. The user can control the SR emission mechanism via dedicated production thresholds.

The new feature is illustrated in Figure 6, showing the photon flux in a bending magnet of a $1.5 \mathrm{TeV}$ muon storage ring. The two plots compare different FLUKA simulations with and without SR emission by decay electrons. In the latter case, without the new 
emission model, the photon flux in the vacuum chamber, shielding and magnet is exclusively due to the impact of decay electrons on the machine aperture. The dipole field bends all decay electrons towards the center of the ring, which leads to a localized energy deposition hot spot on one side of the magnet. In the other case, SR photons are generated along the electron trajectories and can impact either on the inner or outer side of the vacuum chamber, resulting in a larger spread of the energy released in the decay of beam particles. At such or even higher energies, neglecting this phenomenon may lead to a non-faithful representation of the secondary particle distribution in the magnets, energy deposition and background to experiments.

\section{FLAIR, THE FLUKA USER INTERFACE}

\subsection{Flair Version 3 Upgrade}

The FLUKA advanced graphical interface Flair ${ }^{6}$ [22] made its first appearance in 2007. It is a fully integrated working environment that assists the user in all stages of a Monte Carlo simulation, from constructing the input and the geometry to running the simulation, processing the output files and plotting the results. Thanks to its advanced capabilities and intuitive use, it quickly became the most popular way of working with FLUKA. Flair is in constant evolution, continuously expanding its features.

Up to version 2, Flair was based on Python v2, which the Python community decided to phase out in $2020^{7}$. Profiting from this opportunity, it was decided not only to migrate to v3, but also to restructure the code so as to facilitate its further cooperative development. The most notable improvement concerns the decoupling of the graphical interface from the underlying functionality, which was achieved by the introduction of an intermediate abstract layer allowing Flair to support other simulation packages besides FLUKA. While support for MCNP inputs [23] is partial, in the case of Geant4 it is in a quite advanced state, thanks to a dedicated Geant 4 application called Moira (see Section 6).

Particular attention has been paid to computational performance, to further optimize Flair both in terms of CPUtime and numerical precision. A considerable speedup (up to a factor 10) has been attained during the loading and displaying of large input files, thanks to the use of a tk:Text module replacing the previous use of a tk:Canvas ${ }^{8}$. Likewise, the performance and the numerical precision of the geometry editor has been greatly optimized. This has been achieved through the use of $\mathrm{z}$-buffers for $3 \mathrm{D}$ rendering, the implementation of an adaptive anti-aliasing algorithm, and the storage of quadratic surfaces also in reduced form, in order to minimize numerical rounding errors introduced by geometric transformations, to name but a few.

Among the new features of Flair v3, we highlight the graphical tool for planning radiation protection interventions: starting

${ }^{6}$ https://flair.cern

${ }^{7}$ See https://www.python.org/doc/sunset-python-2/

${ }^{8}$ https://www.tcl.tk from a FLUKA Cartesian 3D dose map, the user can define a path which an operator will follow in space and time by means of a spline to obtain a report detailing the dose acquired along the path. In addition, short animated movies can be created displaying the $3 \mathrm{D}$ geometry (and the simulation results) by means of a camera object moving along a user-defined 3D spline. To reinforce the consistency of the input interpretation by FLUKA and Flair, the Flair input file now persistently embeds all the FLUKA input information, which can still be directly manipulated in the FLUKA input file.

\subsection{Flair Developments for Medical Applications}

As high-throughput computational power is getting more accessible, the use of Monte Carlo-based treatment planning systems (TPS) becomes a viable option for clinical applications of radiation therapy. Codes like FLUKA have important advantages over classical treatment planning systems as they can take a wide range of physical effects (e.g., full secondary particle generation due to implants) accurately into account. Yet, the best physics results cannot be fully exploited if they are not put into an anatomic context which speaks to the medical practitioner.

A dedicated effort has been invested in making Flair v3 a selfcontained working environment for the use of FLUKA in medical physics applications. Recent developments include an improved DICOM handling ${ }^{9}$, facilitating the generation of voxel geometries with built-in automatic conversion from Hounsfield units to material composition. For instance, boolean operations can now be applied on predefined regions of interest (ROI) to customize the material assignment in the simulation. Moreover, Flair now includes a DICOM editor to perform common tasks like anonymizing patient information and, in general, the editing of DICOM tags. The embedded RTViewer helps the user to compare the planned dose calculated by a treatment planning system with the dose simulated by FLUKA. An automatic dose-volume-histogram generator (DVH) is included. RTPLAN-modality DICOM files can be imported to generate the corresponding primary-source cards for FLUKA. Furthermore, RTDOSE-modality DICOM files can be converted to FLUKA's standard 3D mesh scoring (USRBIN), which can not only be readily visualized in 2D and 3D in Flair, but also be used as a volumetric primary source for further simulations. The opposite conversion from USRBIN to RTDOSE is also implemented.

While support for $3 \mathrm{D}$ visualization is becoming increasingly available in the domain of Monte Carlo transport codes, visual quality often trails behind current industry standards. In addition, common surface rendering techniques are not suitable for medical visualization due to the loss of contextual information in one dimension. This stems from the fact that there is no well-defined geometry but the input is a discrete cloud of density values obtained

${ }^{9}$ Details concerning the current version of the DICOM standard are available at https://www.dicomstandard.org/current. 

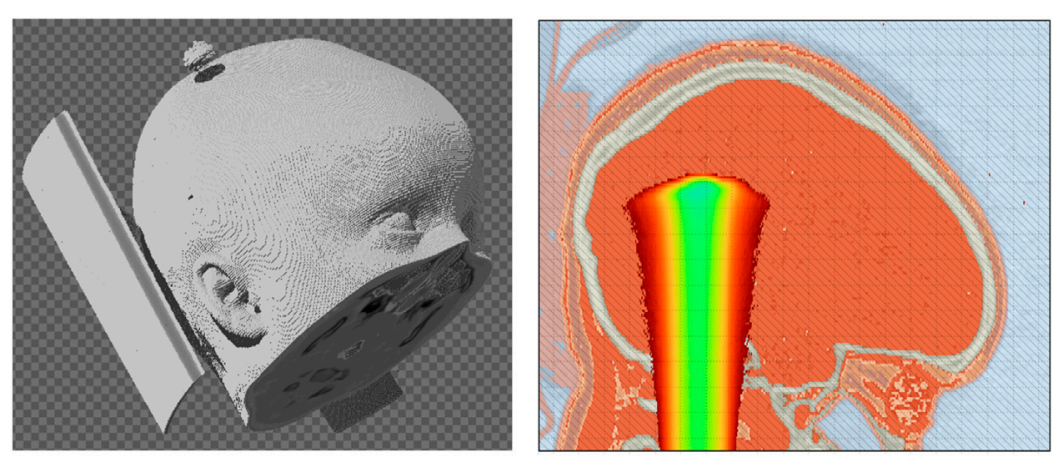

FIGURE 7 | Classic surface-based visualization of a voxelized phantom and dose distribution from an exemplary photon beam irradiation.

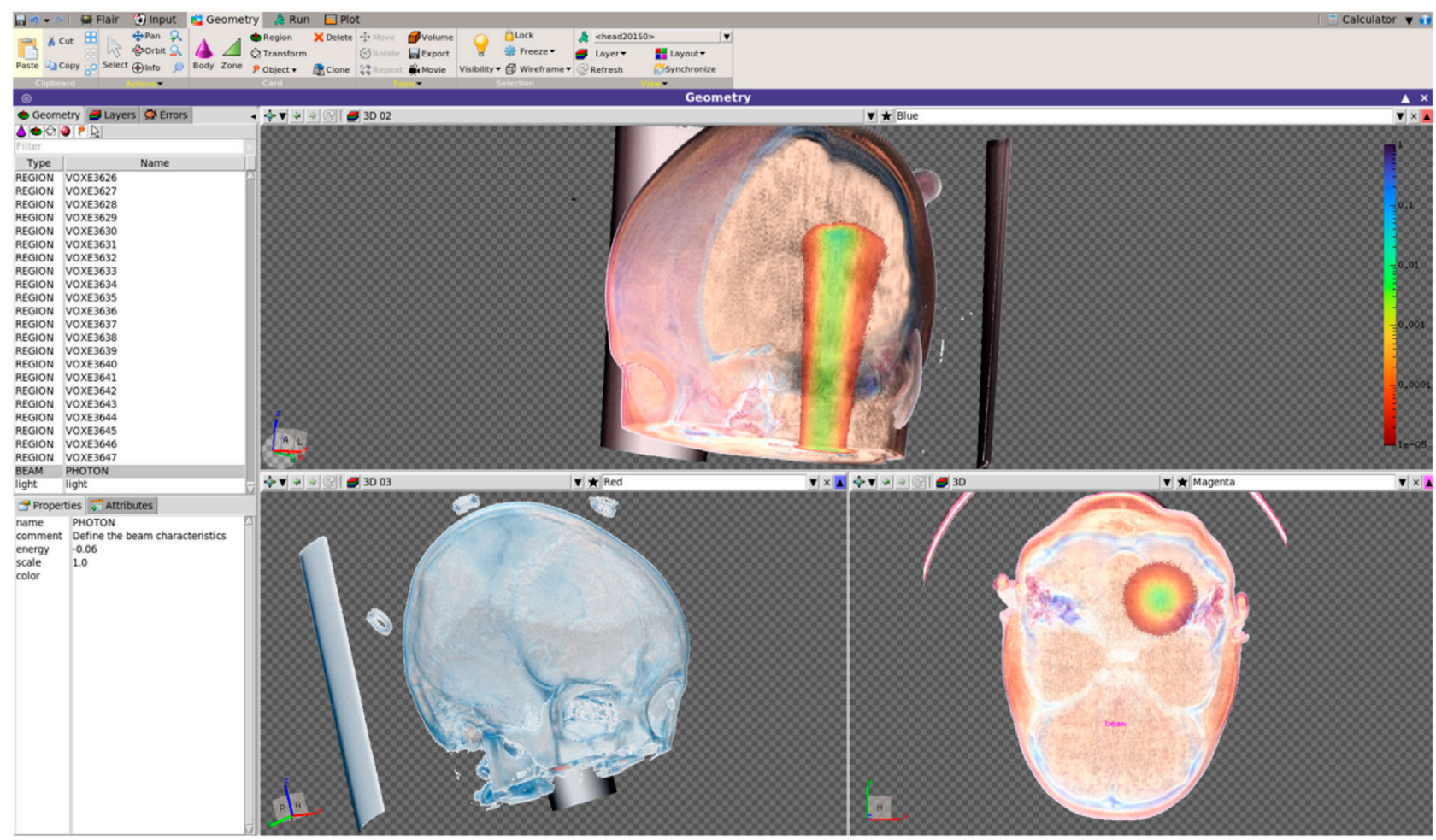

FIGURE 8 | Volumetric visualization of a voxelized phantom and dose distribution from a photon beam irradiation with the new renderer available in Flair v3.

from CT or MRI scans. Consequently, there is no defined geometric surface that one can work with nor are the data continuous in space due to the discretization originating from the data acquisition. The most common way is to assign each point a proxy geometry, e.g. a small cube, and render these with standard techniques. However, this leads to the loss of crucial information as intricate 3D anatomic relationships are not presented anymore to the attending physician. To overcome this drawback, the completely new visualization module in Flair v3 now includes a number of volume rendering techniques for medical applications. These methods allow for proper reconstruction of a 3D function from the discrete dataset via raymarching based algorithms. Texture-based slicing methods would offer performance advantages due to the possibility of GPU implementation. Nonetheless, for large data sets one quickly reaches the limits of standard consumer hardware and, in addition, ray-casting methods seamlessly integrate with Flair's new photorealistic, interactive ray tracer for standard geometries. Figures 7 and 8 illustrate the use of standard surface visualization methods versus Flair v3's new medical visualization module using the same dataset. As can be seen, volumetric rendering retains 3D anatomic structures from the scans even on a very fine level and permits a superior evaluation of the dose distribution in a voxel geometry by the medical physicist or physician. Visualization of the geometry is immediate with progressive refinement based on so-called space 


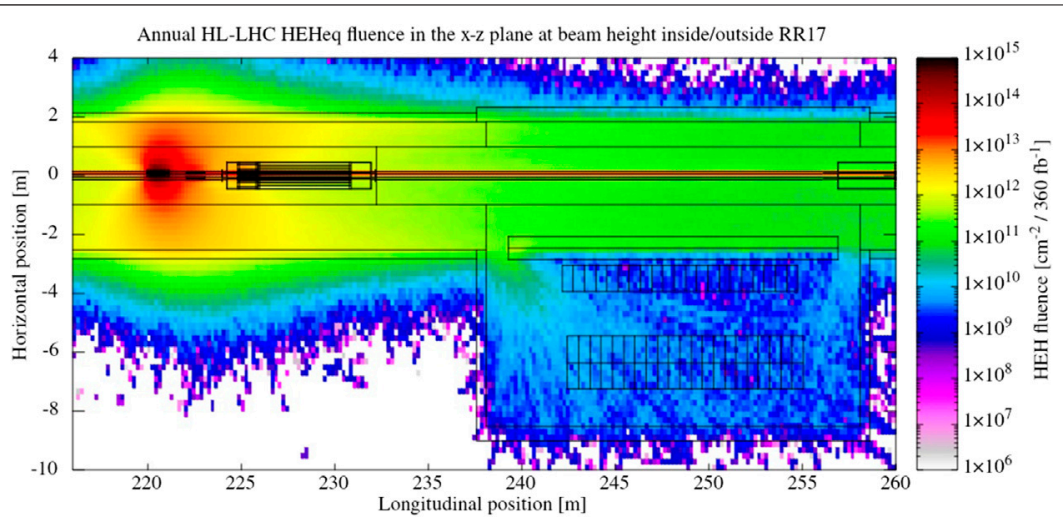

FIGURE 9 | Top view of the annual High Energy Hadron-equivalent fluence at beam height in a portion of the LHC Long Straight Section at Point 1, showing the main tunnel and the nearby RR17 alcove where electronic equipment is hosted.

filling curves opening up real-time user feedback while profiting from optimized use of CPU cache lines.

\section{RADIATION TO ELECTRONICS}

Radiation effects in electronic components and systems are a well-known threat for the reliable operation of electronics in space and can also be a risk for the successful operation of high energy accelerators [24]. In fact, during the LHC Run 1 (2010-2013), Radiation to Electronics (R2E) issues were one of the main causes of unavailability and downtime of the machine [25]. Monte Carlo transport codes constitute a powerful tool to predict and mitigate such effects, both with regards to the simulation of the radiation environment as well as to its effects on electronics. As will be shown, FLUKA is critical in the context of the R2E activities, where it is used for a variety of purposes and applications.

\subsection{High Energy Accelerator Radiation Environment}

A key task carried out with FLUKA is the simulation of the radiation field in the CERN accelerator complex, as well as in experimental and test facilities. To this end, FLUKA is used to calculate $3 \mathrm{D}$ space distributions of the key radiation level quantities that are needed to assess radiation impact on electronic equipment, both in terms of lifetime degradation and stochastic SEEs.

Lifetime effects are quantified with Total Ionising Dose (TID), i.e., the energy loss due to ionising radiation per unit mass, and the equivalent fluence of $1 \mathrm{MeV}$ neutrons in Silicon, which is used to express Displacement Damage effects in material lattices. On the other hand, FLUKA provides the High Energy Hadronequivalent (HEH-eq) fluence, i.e., the fluence of all hadrons with $E>20 \mathrm{MeV}$ plus the neutrons in the $0.2-20 \mathrm{MeV}$ range with energy-dependent Weibull weights, and the thermal neutron equivalent fluence, where neutrons are weighted proportionally to the inverse of their velocity. These quantities are directly linked to the SEE evaluation.
Figure 9 shows a representative example of radiation environment characterization. It displays the $\mathrm{HEH}$-eq fluence in a portion of LHC tunnel located between 216 and $260 \mathrm{~m}$ from Interaction Point (IP) 1, where beam collisions take place. In this case, FLUKA is employed to generate and transport products of inelastic proton-proton interactions from IP1, inducing radiation showers through machine elements, the LHC tunnel infrastructure and nearby underground areas. This way the space distribution of HEH-eq fluence can be obtained and 1D and 2D projections can be extracted for an area of interest (as shown in Figure 9), which in this case consists of the highlighted tunnel section and the nearby RR17 alcove hosting electronic equipment. The simulation is scaled to an integrated luminosity of $360 \mathrm{fb}^{-1}$, so as to represent annual radiation levels in the final years of LHC operation after the High-Luminosity LHC upgrade. These results, along with similar calculations in other LHC areas, serve as basis for the definition of radiation level specifications for electronics. They are essential to define qualification requirements in accordance with a standard Radiation Hardness Assurance procedure [26].

\subsection{Monte Carlo Simulation of Single Event Effects}

As introduced above, radiation effects can be classified as either cumulative or stochastic. Monte Carlo codes are particularly suited to the prediction and better understanding of the latter which are of random and single-event-like nature. This capability is especially relevant for high energy accelerator applications in which the related response to the passage of a broad range of particles and energies [27] needs to be derived from a relatively limited data set, typically consisting of experimental results from $200 \mathrm{MeV}$ protons.

In particular, codes like FLUKA can accurately simulate energy deposition distributions from single-particle events in sensitive volumes (SV) of microelectronic components. After the energy deposition step, additional dedicated simulations are performed to determine charge collection and related circuit response, which in turn are used to predict the SEE 


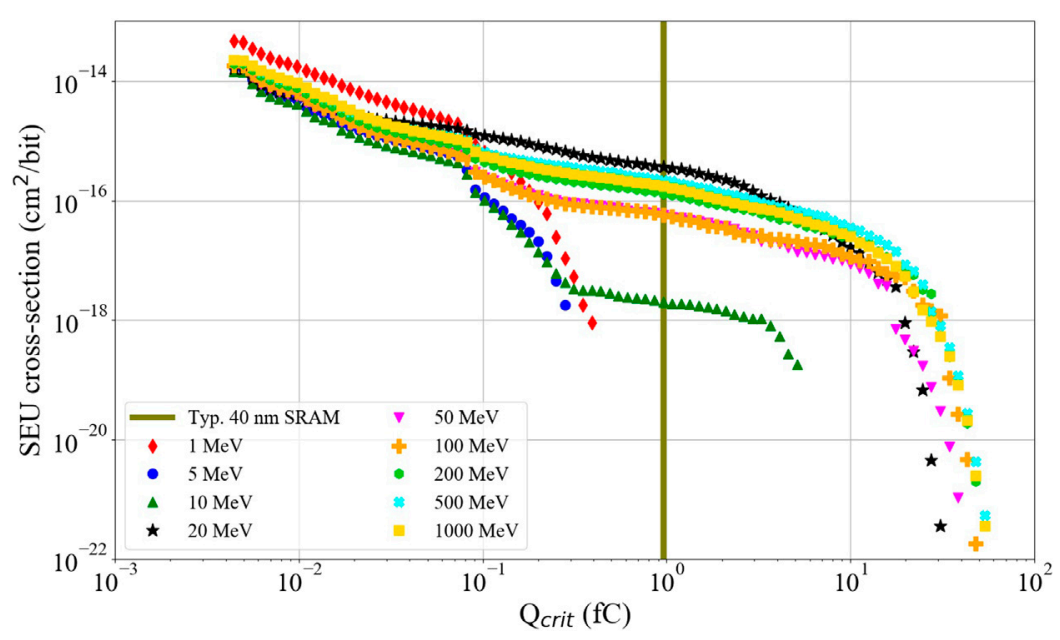

FIGURE 10 | FLUKA simulation of SEU cross section obtained from the integral of the energy deposition distribution by mono-energetic photons above $Q_{\text {crit }}$ in the RPP model representing an SRAM sensitive volume for SEU, plotted as a function of $Q_{\text {crit. }}$ The vertical line represents a typical value of the critical charge for 40 nm devices.

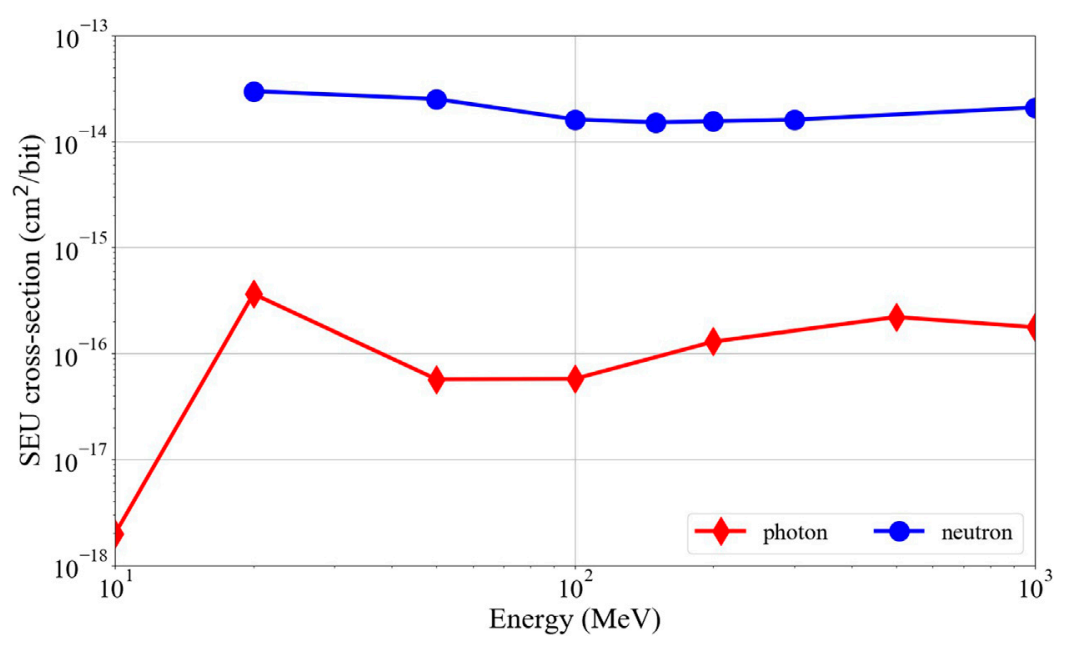

FIGURE 11 | Simulated photon and neutron SEU cross section for 40 nm commercial SRAM.

cross section of the device. In first approximation however, FLUKA can be used for this purpose as well, modelling the SV of the device as a Rectangular Parallelepiped (RPP) [28] or Integral RPP (IRPP)[29], assuming the energy deposited in this volume directly translates to ionization charge collected by the circuit with $100 \%$ efficiency. Under this assumption, an SEE will occur if the collected charge is above a certain critical charge value $\left(Q_{c r i t}\right)$ in the RPP case or typically determined experimentally through heavy ion SEE testing, in the IRPP case.

This method has been applied to demonstrate that all hadrons have an approximately equal SEE cross section above $20 \mathrm{MeV}$ [30] in a mixed-field environment. For highly sensitive devices (i.e., low $Q_{\text {crit }}$ with small SVs, this finding has been validated within a factor 2 up to hundreds of $\mathrm{GeV}$ [31]. The resulting SEE rate prediction is typically estimated within $\pm 50 \%$, which is considered acceptable for mixed radiation field applications. Moreover, the related predictions were successfully benchmarked against experimental measurements, [30].

Various exceptions to the above formalism were studied with FLUKA. This includes for example differences between pion- and nucleon-induced SEE cross section [32], the enhanced SEE cross section of low-sensitivity devices (i.e. high $Q_{c r i t}$ with high- $Z$ material near the SV in the hadron energy range from $200 \mathrm{MeV}$ to $3 \mathrm{GeV}$ due to fission reactions [33] and the impact of directly ionizing light charged particles such as protons [34] and muons [35]. Additional studies investigated SEEs induced by electro-and photonuclear reactions [36] and, via a Geant4-based extension, by neutrons in the $0.1-10 \mathrm{MeV}$ neutron range [37].

Beyond accelerator applications, similar FLUKA models were used to predict the dominance of protons versus heavy ions for 
nuclear induced events in space [38], as well as to use experimental SEE results obtained in hadron environments to predict or bound the error rate induced by heavy ions [39]. Likewise, FLUKA was used to study fragmentation and indirect energy deposition from high-energy heavy ions [40].

As a final illustration of the FLUKA capabilities to gauge radiation effects on electronics, the following lines present results of the simulation of SEEs induced by mono-energetic photons in the $1 \mathrm{MeV}-1 \mathrm{GeV}$ range in an RPP model of a $40 \mathrm{~nm}$ commercial SRAM ${ }^{10}$. The SEE cross section is obtained from the integral of the distribution of the energy deposited by the photons in the SV above a fixed threshold referred to as $Q_{\text {crit }}$, assuming $Q[\mathrm{fC}]=\mathrm{E}_{\mathrm{dep}}[\mathrm{GeV}] \times 10^{6} / 22.5$ in Silicon. Its simulated value is shown in Figure $\mathbf{1 0}$ as a function of $Q_{\text {crit }}$. Considering a critical charge of $1 \mathrm{fC}$, Figure $\mathbf{1 0}$ shows that photons below $10 \mathrm{MeV}$ are not capable of inducing SEUs. Indeed, they interact mostly through Compton scattering which deposits low energy in the SV.

Over $10 \mathrm{MeV}$, photo-nuclear reactions lead to higher energy deposition values which can trigger SEUs. The photon-induced SEU cross section is represented in Figure $\mathbf{1 1}$ as a function of the photon energy and compared to the neutron-induced one, in both cases for $Q_{\text {crit }}=1 \mathrm{fC}$. This shows, as expected, that the latter is higher by roughly two orders of magnitude. Still, for specific radiation environments such as those generated by high energy electrons (e.g., downstream the CLEAR beam dump [41], absorbing a beam of $200 \mathrm{MeV}$ electrons) the relative proportion of high energy photons can be prevalent, hence potentially having a significant or even dominant contribution to the overall SEU rate.

\section{CODE TESTING AND BENCHMARKING}

Ensuring the integrity and quality of FLUKA throughout its full software lifecycle is of utmost importance. A software quality assurance plan is being redefined and put in place accordingly. It implements best practices in software development tailored to the specific needs of a Monte Carlo radiation transport simulations code. The quality assurance plan now includes modern source code management, a dedicated testing framework and initiatives to produce experimental data for benchmarks. This is complemented by a feedback reporting mechanism via the user forum ${ }^{11}$ that generates input for the development process.

\subsection{Source Code Management and Continuous Integration}

The FLUKA source code lifecycle is managed using the CERN GitLab instance ${ }^{12}$. The latter offers a state-of-the-art infrastructure for full version control based on the git

\footnotetext{
${ }^{10}$ detailed in [34].

${ }^{11}$ https://fluka-forum.web.cern.ch/

${ }^{12} \mathrm{https} / /$ gitlab.cern.ch
}

version control system [42] as well as Continuous Integration (CI). A continually growing number of test cases is included in the CI framework, aimed both at probing new development features and ensuring the consistency with respect to the established FLUKA version. These test cases run automatically for every pushed commit. A further in-depth validation at the model level and for fullscale test cases is performed within the FLUKAVAL framework (see next subsection). As an additional quality assurance check prior to public releases, the code is deployed and intensively tested in the respective computing clusters of the collaborating institutes, monitoring its performance and stability in real-world applications.

\subsection{FLUKAVAL Framework}

The FLUKAVAL framework is a Python-based command line application that enables semi-automatic batch submission, processing, validation and reporting of test cases. Any FLUKA input and even model-level based tests can be adapted and integrated in a few steps into the framework. FLUKAVAL makes extensive use of the git version control system to store simulation and reference data sets on the CERN GitLab instance. In its current version, FLUKAVAL is optimised for the submission of a large number of test cases to the CERN Batch Service (LXBATCH), which provides distributed computing power with over 220,000 CPU cores.

FLUKA output data are stored in standardised JSON format, which is compatible with the Geant Validation Portal ${ }^{13}$, to simplify future direct comparisons of identical test cases with Geant4.

The results are processed, validated and compiled in a comprehensive evaluated report, which allows the direct comparison of results from the FLUKA version under validation with results obtained with previous FLUKA versions, other radiation transport codes and/or experimental data. A quantitative analysis is performed in addition to a qualitative comparison whenever possible. The resulting report is stored in the CERN document management system once the proper approval process is positively completed.

\subsection{Validation Procedure}

One of the ways to validate a FLUKA version and achieve quality assurance is to directly and quantitatively compare results from the version under development to results obtained with previous released versions of the code. A growing number of test cases is available within the FLUKAVAL framework, each of them aiming to investigate selected features of the code. Specific acceptance criteria are chosen for each of the test cases based on the observable/physics quantity to validate.

Two kinds of test cases are available at present in order to validate the different features of the code, namely on the model/interaction level and full-scale test cases. The first kind of test cases aims to validate a FLUKA version at the

\footnotetext{
${ }^{13}$ https://geant-val.cern.ch/
} 
model/interaction level by running specific drivers that have access to internal functionalities of the code. Among others, these drivers allow to estimate reaction cross sections, double differential cross sections and yields as a function of energy for any kind of projectile and known target element either as a single isotope or in its natural isotopic composition. These tailored test cases provide a fine control over the changes and improvements of the implemented physics model. Full-scale test cases, instead, aim to validate the code with FLUKA simulations based on real case applications. These test cases provide a coverage of different fields of application and FLUKA functionalities. They can test several observables such as energy deposition, energy and/or spatial particle fluence distributions, radionuclide inventory and residual dose in a more elaborate case. That can include, for example, complex geometries and material definitions, tracking in magnetic fields, special sources and custom user routines.

Comparisons to other codes also provide useful input for the development process. The FLUKAVAL framework can include simulation results of other Monte Carlo radiation transport simulations codes and compare them quantitatively to FLUKA results. In addition, the FLUKA code is regularly included in international Monte Carlo code inter-comparisons, for example the particle production inter-comparison at the SATIF workshop series [43, 44].

Comparisons to experimental data are vital for the quality assurance of FLUKA. The FLUKAVAL framework can embed experimental data and allows for a quantitative comparison with FLUKA results. The experimental data in the FLUKAVAL framework ranges from the single interaction level data, e.g., radionuclide production cross-sections from the EXFOR database, up to measurements in large experiments.

All the above is complemented by a long-standing initiative to generate new experimental data for benchmarks of radiation transport simulation codes with a focus on the relevance to real-world applications. Examples are neutron spectra at the CERN-EU High-Energy Fields (CERF) facility [45-47], neutron spectra and activation at the CERN Shielding Benchmark Facility (CSBF) [48, 49], activation at the CERN High-energy AcceleRator (CHARM) test facility [50] and activation due to stray radiation from the annihilation of lowenergy antiprotons in steel [51].

\section{OUTLOOK}

The present code has reached a high level of maturity, but its rigid structure and procedural programming paradigm pose an intrinsic limitation for future contributions and developments. While improving and extending the physics performance of FLUKA remains a key objective, a concurrent development activity has been started, aiming at a substantial evolution of the code architecture. Modernizing the codebase, increasing its modularity, and reinforcing a collaborative environment is an essential path for assuring the long-term sustainability of FLUKA. The new code will be based on $\mathrm{C}++$, profiting from the advantages of an objectoriented design. At the same time, key objectives are to improve the user friendliness and to offer a rich set of built-in options beyond the existing functionality of present FLUKA releases.

\subsection{Moira}

The first stage of the development project, which is named Moira, is presently in an exploratory phase. To foster synergies with other Monte Carlo codes and benefit from existing development efforts, the new code will be based on the Geant 4 framework [52-54]. The technical functionalities of FLUKA are translated into a Geant4-based application, incorporating a wide spectrum of existing FLUKA capabilities (e.g., scoring, biasing, radioactive decay calculations). Where available, existing features of Geant 4 will be utilized.

The new code will continue to support combinatorial geometries based on the present FLUKA input format, using Flair as an external geometry navigator. At the same time, it will offer access to Geant4-compatible geometry descriptions, for example in the GDML format. A versatile set of built-in scoring options will be provided, which can be used with any type of geometry description. Moira will be closely integrated with Flair in order to preserve an intuitive visual user experience through a graphical user interface and an advanced geometry viewer.

\subsection{A Fifth FLUKA Generation}

In a second stage, the FLUKA physics models will be migrated. When the Moira program reaches a sufficient level of maturity, it will be distributed as a new FLUKA generation. The goal is to provide a fully integrated package to the user with a fixed set of physics models that have undergone strict quality assurance processes and thus will deliver well identifiable physics results, being consistent with the ones of FLUKA v4 at that time. The development effort until the first release is expected to expand over several years. Nevertheless, in the interest of the Monte Carlo user community, it is planned to distribute intermediate Moira versions at earlier stages, enabling the use of the FLUKA input files also for Geant 4 calculations. The modernization and modularization of the program, profiting in particular from existing interfaces in Geant4, will facilitate contributions from a wider community.

\section{DATA AVAILABILITY STATEMENT}

The raw data supporting the conclusions of this article will be made available by the authors, without undue reservation.

\section{AUTHOR CONTRIBUTIONS}

The authors of this manuscript are participants in the FLUKA.CERN Collaboration. Section 1 was written by FC. 
DC, LE, AL, FSP, and PS elaborated section 2. CT and VV composed section 3. ACo, RGA and GL produced section 4. CA, $\mathrm{DB}, \mathrm{RF}, \mathrm{AI}$, and MW wrote section 5 , while $\mathrm{GH}$ and AL provided section 6. NC, DH, FOR, SR, AT, RV, AW have revised the manuscript. All authors contributed to the work and approved the submitted version.

\section{REFERENCES}

1. Battistoni G, Boehlen T, Cerutti F, Chin PW, Esposito LS, Fassò A, et al. (2015). Overview of the FLUKA Code. Ann Nucl Energ 82:10-8. doi:10.1016/ j.anucene.2014.11.007

2. Ambrosino F, Roca V, Buompane R, Sabbarese C (2020). Development and Calibration of a Method for Direct Measurement of 220rn (Thoron) Activity Concentration. Appl Radiat Isot 166:109310. doi:10.1016/ j.apradiso.2020.109310

3. Bielajew A (1987). Electron Transport in E and B fields. In: Monte Carlo Transport of Electrons and Photons. New York, NY: Plenum Press. p. 421-34.

4. Afonin AG, Baranov VT, Biryukov VM, Breese MBH, Chepegin VN, Chesnokov YA, et al. (2001). High-efficiency Beam Extraction and Collimation Using Channeling in Very Short Bent Crystals. Phys Rev Lett 87:94802. doi:10.1103/physrevlett.87.094802

5. Scandale W, Arduini G, Butcher M, Cerutti F, Gilardoni S, Lechner A, et al. (2014). Observation of Focusing of $400 \mathrm{GeV} / \mathrm{c}$ Proton Beam with the Help of Bent Crystals. Phys Lett B 733:366-72. doi:10.1016/ j.physletb.2014.05.010

6. Rossi RThe UA9 collaboration (2016). Observation of Channeling in Bent Crystals at the CERN LHC. In: 38th International Conference on High Energy. PoS ICHEP2016, Chicago. p. 867. doi:10.22323/1.282.0867

7. Rossi R (2017). Experimental Assessment of Crystal Collimation at the Large Hadron Collider. Ph.D. Thesis. Presented 26 Jan 2018.

8. Redaelli S, Butcher M, Barreto C, Losito R, Masi A, Mirarchi D, et al. (2021). First Observation of Ion Beam Channeling in Bent Crystals at Multi-Tev Energies. Eur Phys J C 81:1-7. doi:10.1140/epjc/s10052-02108927-x

9. Schoofs P, Cerutti F, Ferrari A, Smirnov G (2015). Benchmark of the FLUKA Model of crystal Channeling against the UA9-H8 experiment. Nucl Instr Methods Phys Res Section B: Beam Interactions Mater Atoms 355:374-7. doi:10.1016/j.nimb.2015.03.074

10. Esbensen H, Fich O, Golovchenko JA, Madsen S, Nielsen H, Schiøtt HE, et al. (1978). Random and Channeled Energy Loss in Thin Germanium and Silicon Crystals for Positive and Negative 2-15-GeV/cpions, Kaons, and Protons. Phys Rev B 18:1039-54. doi:10.1103/PhysRevB.18.1039

11. Schoofs P (2014). Monte Carlo Modeling of Crystal Channeling at High Energies. Ph.D. Thesis. CERN-THESIS-2014-131.

12. Alliot C, Audouin N, Barbet J, Bonraisin AC, Bosse VR, Bourdeau CC, et al. (2015). Is There an Interest to Use Deuteron Beams to Produce Nonconventional Radionuclides? Front Med 2:31. doi:10.3389/fmed.2015.00031

13. Sitarz M, Szkliniarz K, Jastrzębski J, Choiński J, Guertin A, Haddad F, et al. (2018). Production of Sc Medical Radioisotopes with Proton and Deuteron Beams. Appl Radiat Isot 142:104-12. doi:10.1016/ j.apradiso.2018.09.025

14. Takada M, Mihara E, Sasaki M, Nakamura T, Honma T, Kono K, et al. (2004). Neutron Field Produced by $25 \mathrm{MeV}$ Deuteron on Thick Beryllium for Radiobiological Study; Energy Spectrum. Radiat Prot Dosimetry 110:601-6. doi:10.1093/rpd/nch142

15. Chauvin N, Akagi T, Bellan L, Beauvais P-Y, Bolzon B, Cara P, et al. (2019). Deuteron Beam Commissioning of the Linear IFMIF Prototype Accelerator Ion Source and Low Energy Beam Transport. Nucl Fusion 59:106001. doi:10.1088/1741-4326/ab1c88

16. Serber R (1947). The Production of High Energy Neutrons by Stripping. Phys Rev 72:1008-16. doi:10.1103/physrev.72.1008

17. Ferrari A, Sala P (1998). The Physics of High Energy Reactions. In: Proceeding: Workshop on Nuclear Reaction Data and Nuclear Reactor. Physics, Design and Safety, Trieste (Italy). World Scientific. 424.

\section{ACKNOWLEDGMENTS}

We would like to deeply thank the CERN Knowledge Transfer and Legal Service teams for their essential and extended support. Our appreciation also goes to the FLUKA.CERN Collaboration Board members for their strong commitment.

18. Baur G, Rösel F, Trautmann D (1976). Investigations of the Deuteron BreakUp on Heavy Nuclei above the Coulomb Barrier. Nucl Phys A 265:101-12. doi:10.1016/0375-9474(76)90118-4

19. Titus L (2016). Effects of Non-locality on Transfer Reactions. Ph.D. Thesis. East Lansing, MI: Michigan State University. arXiv:1604.00094v1.

20. Cerutti F, Battistoni G, Capezzali G, Colleoni P, Ferrari A, Gadioli E, et al. (2006). Low Energy Nucleus-Nucleus Reactions: the BME Approach and its Interface with FLUKA. In: Proceedings of the 11th International Conference on Nuclear Reaction Mechanisms, Varenna.

21. Ridikas D, Mittig W, Savajols H, Roussel-Chomaz P, Foertsch S, Lawrie J, et al. (2000). Inclusive Proton Production Cross Sections in (D,xp) Reactions Induced by $100 \mathrm{MeV}$ Deuterons. Phys Rev C 63:014610. doi:10.1103/ physrevc.63.014610

22. Vlachoudis V (2009). FLAIR: A Powerful but User Friendly Graphical Interface for FLUKA. In: Proceedings: International Conference on Mathematics, Computational Methods and Reactor Physics, Saratoga Springs, NY.

23. Werner CJ (2017). MCNP Users Manual-Code Version 6.2. Los Alamos, NM: Los Alamos National Security LLC.

24. García Alía R, Brugger M, Danzeca S, Cerutti F, de Carvalho Saraiva JP, Denz R, et al. (2017). Single Event Effects in High-Energy Accelerators. Semiconductor Sci Techn 32:034003. doi:10.1088/1361-6641/aa5695

25. Apollonio A, Brugger M, Rossi L, Schmidt R, Todd B, Wollmann D, et al. (2015). Roadmap towards High Accelerator Availability for the CERN HLLHC Era. In: Proceedings, 6th International Particle Accelerator Conference (IPAC 2015). Richmond, Virginia, USA.

26. Aberle O, Béjar Alonso I, Brüning O, Fessia P, Rossi L, Tavian L, et al. (2020). HighLuminosity Large Hadron Collider (HL-LHC): Technical Design Report. CERN Yellow Reports: Monographs. Geneva: CERN. doi:10.23731/CYRM-2020-0010

27. Alia RG, Tali M, Brugger M, Cecchetto M, Cerutti F, Cononetti A, et al. (2020). Direct Ionization Impact on Accelerator Mixed-Field Soft-Error Rate. IEEE Trans Nucl Sci 67:345-52. doi:10.1109/TNS.2019.2951307

28. Weller RA, Reed RA, Warren KM, Mendenhall MH, Sierawski BD, Schrimpf RD, et al. (2009). General Framework for Single Event Effects Rate Prediction in Microelectronics. IEEE Trans Nucl Sci 56:3098-108. doi:10.1109/ tns.2009.2033916

29. Petersen EL, Pouget V, Massengill LW, Buchner SP, McMorrow D (2005). Rate Predictions for Single-Event Effects - Critique II. IEEE Trans Nucl Sci 52: 2158-67. doi:10.1109/TNS.2005.860687

30. García Alía R, Biskup B, Brugger M, Calviani M, Poivey C, Roed K, et al. (2013). SEU Measurements and Simulations in a Mixed Field Environment IEEE Trans Nucl Sci 60:2469-76. doi:10.1109/TNS.2013.2249096

31. García Alía R, Brugger M, Danzeca S, Ferlet-Cavrois V, Poivey C, Roed K, et al. (2013). SEE Measurements and Simulations Using Mono-Energetic GeVEnergy Hadron Beams. IEEE Trans Nucl Sci 60:4142-9. doi:10.1109/ TNS.2013.2279690

32. Coronetti A, García Alía R, Cerutti F, Hajdas W, Soderstrom D, Javanainen A, et al. (2021). The Pion Single-Event Latch-Up Cross Section Enhancement: Mechanisms and Consequences for Accelerator Hardness Assurance. IEEE Trans Nucl Sci 68:1613-22. doi:10.1109/TNS.2021.3070216

33. García Alía R, Brugger M, Cecchetto M, Cerutti F, Danzeca S, Delrieux M, et al. (2018). SEE Testing in the 24-GeV Proton Beam at the CHARM Facility. IEEE Trans Nucl Sci 65:1750-8. doi:10.1109/TNS.2018.2829916

34. Coronetti A, García Alía R, Wang J, Tali M, Cecchetto M, Cazzaniga C, et al. (2021). Assessment of Proton Direct Ionization for the Radiation Hardness Assurance of Deep Submicron Srams Used in Space Applications. IEEE Trans Nucl Sci 68:937-48. doi:10.1109/TNS.2021.3061209

35. Infantino A, García Alía R, Brugger M (2017). Monte Carlo Evaluation of Single Event Effects in a Deep-Submicron Bulk Technology: Comparison 
between Atmospheric and Accelerator Environment. IEEE Trans Nucl Sci 64: 596-604. doi:10.1109/TNS.2016.2621238

36. Tali M, García Alía R, Brugger M, Ferlet-Cavrois V, Corsini R, Farabolini W, et al. (2019). Mechanisms of Electron-Induced Single-Event Latchup. IEEE Trans Nucl Sci 66:437-43. doi:10.1109/TNS.2018.2884537

37. Cecchetto M, García Alía R, Wrobel F, Coronetti A, Bilko K, Lucsanyi D, et al. (2021). 0.1-10 MeV Neutron Soft Error Rate in Accelerator and Atmospheric Environments. IEEE Trans Nucl Sci 68:873-83. doi:10.1109/TNS.2021.3064666

38. García Alía R, Brugger M, Ferlet-Cavrois V, Brandenburg S, Calcutt J, Cerutti F, et al. (2017). Proton Dominance of Sub-LET Threshold GCR SEE Rate. IEEE Trans Nucl Sci 64:388-97. doi:10.1109/TNS.2016.2628363

39. García Alía R, Brugger M, Daly E, Danzeca S, Ferlet-Cavrois V, Gaillard R, et al. (2017). Simplified SEE Sensitivity Screening for COTS Components in Space. IEEE Trans Nucl Sci 64:882-90. doi:10.1109/TNS.2017.2653863

40. Kastriotou M, Fernandez-Martinez P, García Alía R, Cazzaniga C, Cecchetto M, Coronetti A, et al. (2020). Single Event Effect Testing with Ultrahigh Energy Heavy Ion Beams. IEEE Trans Nucl Sci 67:63-70. doi:10.1109/ TNS.2019.2961801

41. Sjobak K, Adli E, Bergamaschi M, Burger S, Corsini R, Curcio A, et al. (2019). Status of the clear Electron Beam User Facility at Cern. In: Proceedings of the 10th International Particle Accelerator Conference, Melbourne, Australia.

42. Chacon S, Straub B (2014). Pro Git. Apress.

43. Hirayama H, Sanami T (2014). Inter-Comparison of Particle Production. In: Shielding Aspects of Accelerators, Targets and Irradiation Facilities (SATIF12); 28-30 April, 2014; Batavia, Illinois, USA. 242-51. OECD/NEA/NSA/ $\mathrm{R}(2015) 3$.

44. Hirayama H, Sanami T (2018). Inter-Comparison of Particle Production. In: Shielding Aspects of Accelerators, Targets and Irradiation Facilities (SATIF14); 30 October-2 November, 2018; Gyeonju, South Korea.

45. Nakao N, Rokni SH, Brugger M, Roesler S, Vincke H, Kosako K (2006). Calculation of High-Energy Neutron Spectra with Different Monte Carlo Transport Codes and Comparison to Experimental Data Obtained at the CERF Facility. In: Shielding Aspects of Accelerators, Targets and Irradiation Facilities - SATIF 8, Proceedings of the 8th Meeting, Nam-Gu Pohang, Republic of Korea, 2006.

46. Pozzi F, Silari M (2020). The CERN-EU High-Energy Reference Field (CERF) Facility: New FLUKA Reference Values of Spectral Fluences, Present and Newly Proposed Operational Quantities. Nucl Instr Methods Phys Res Section A: Acc Spectrometers Detectors Associated Equipment 979:164477. doi:10.1016/ j.nima.2020.164477

47. Nakao N, Taniguchi S, Roesler S, Brugger M, Hagiwara M, Vincke H, et al. (2008). Measurement and Calculation of High-Energy Neutron Spectra behind Shielding at the CERF 120GeV/c Hadron Beam Facility. Nucl Instr Methods Phys Res Section B: Beam Interactions Mater Atoms 266:93-106. doi:10.1016/ j.nimb.2007.09.043

48. Iliopoulou E, Bamidis P, Brugger M, Froeschl R, Infantino A, Kajimoto T, et al. (2018). Measurements and FLUKA Simulations of Bismuth and Aluminium Activation at the CERN Shielding Benchmark Facility (CSBF). Nucl Instr
Methods Phys Res Section A: Acc Spectrometers Detectors Associated Equipment 885:79-85. doi:10.1016/j.nima.2017.12.058

49. Iliopoulou E, Bamidis P, Brugger M, Froeschl R, Infantino A, Kajimoto T, et al. (2018). Measurements and FLUKA Simulations of Bismuth, Aluminium and Indium Activation at the Upgraded CERN Shielding Benchmark Facility (CSBF). J Phys Conf Ser 1046:012004. doi:10.1088/1742-6596/1046/1/012004

50. Nakao N, Kajimoto T, Sanami T, Froeschl R, Iliopoulou E, Infantino A, et al. (2021). Measurements and Monte Carlo Simulations of High-Energy Neutron Streaming through the Access Maze Using Activation Detectors at $24 \mathrm{Gev} / \mathrm{c}$ Proton Beam Facility of Cern/charm. J Nucl Sci Techn 58:899-907. doi:10.1080/00223131.2021.1887003

51. Ahdida C, Froeschl R, Iliopoulou E, Infantino A, Jensen S (2020). Measurements and FLUKA Simulations of Aluminium, Bismuth and Indium Activation by Stray Radiation from the Annihilation of Low Energy Antiprotons. Nucl Instr Methods Phys Res Section A: Acc Spectrometers Detectors Associated Equipment 950:162972. doi:10.1016/j.nima.2019.162972

52. Agostinelli S, Allison J, Amako K, Apostolakis J, Araujo H, Arce P, et al. (2003). Geant4-a Simulation Toolkit. Nucl Inst Meth A 506:250-303. doi:10.1016/ S0168-9002(03).01368-8

53. Allison J, Amako K, Apostolakis J, Araujo HM, Arce Dubois P, Asai M, et al. (2006). Geant4 Developments and Applications. IEEE Trans Nucl Sci 53: 270-8. doi:10.1109/TNS.2006.869826

54. Allison J, Amako K, Apostolakis J, Arce P, Asai M, Aso T, et al. (2016). Recent Developments in Geant4. Nucl Inst Meth A 835:186-225. doi:10.1016/ j.nima.2016.06.125

Conflict of Interest: The authors declare that the research was conducted in the absence of any commercial or financial relationships that could be construed as a potential conflict of interest.

Publisher's Note: All claims expressed in this article are solely those of the authors and do not necessarily represent those of their affiliated organizations or those of the publisher, the editors, and the reviewers. Any product that may be evaluated in this article, or claim that may be made by its manufacturer, is not guaranteed or endorsed by the publisher.

Copyright (๔ 2022 Ahdida, Bozzato, Calzolari, Cerutti, Charitonidis, Cimmino, Coronetti, D’Alessandro, Donadon Servelle, Esposito, Froeschl, García Alía, Gerbershagen, Gilardoni, Horváth, Hugo, Infantino, Kouskoura, Lechner, Lefebvre, Lerner, Magistris, Manousos, Moryc, Ogallar Ruiz, Pozzi, Prelipcean, Roesler, Rossi, Sabaté Gilarte, Salvat Pujol, Schoofs, Stránský, Theis, Tsinganis, Versaci, Vlachoudis, Waets and Widorski. This is an open-access article distributed under the terms of the Creative Commons Attribution License (CC BY). The use, distribution or reproduction in other forums is permitted, provided the original author(s) and the copyright owner(s) are credited and that the original publication in this journal is cited, in accordance with accepted academic practice. No use, distribution or reproduction is permitted which does not comply with these terms. 